\title{
Pacific
}

Journal of

Mathematics

\section{ENTROPY VERSUS ORBIT EQUIVALENCE FOR MINIMAL} HOMEOMORPHISMS

M. Michael Boyle AND David E. Handelman 


\title{
ENTROPY VERSUS ORBIT EQUIVALENCE FOR MINIMAL HOMEOMORPHISMS
}

\author{
Mike Boyle and David Handelman
}

\begin{abstract}
Every minimal homeomorphism of a Cantor set is strongly orbit equivalent to a homeomorphism of zero entropy. The dyadic adding machine is strongly orbit equivalent to homeomorphisms of all entropies.
\end{abstract}

1. Introduction and definitions. We construct, using the lexicographic or "adic" maps of Vershik (as modified by Herman, Putnam, and Skau [P], [HPS], [Sk]), examples of minimal homeomorphisms of all possible (topological) entropies (including infinite) which are "strongly" orbit equivalent to the dyadic adding machine. In particular, this answers in a decisively negative way the question as to whether orbit equivalent homeomorphisms have the same entropy. We also show that any minimal homeomorphism of the Cantor set is strongly orbit equivalent to one of zero entropy.

We recall some definitions. A source for these is the survey article of Skau [Sk]. A Bratteli diagram $B$ is a directed graph whose vertex set decomposes into finite subsets, "levels" or "rows", $B_{k}(k=$ $0,1, \ldots)$, together with edges from vertices in $B_{k}$ to vertices in $B_{k+1}$; additionally, every vertex of $B_{k}$ is joined to a vertex of $B_{k+1}$. The Bratteli diagram is simple if for all $k$, there exists $k^{\prime}>k$ such that for every vertex $v$ in $B_{k}$ and every vertex $v^{\prime}$ in $B_{k^{\prime}}$, there is a path from $v$ to $v^{\prime}$. The Bratteli diagram is pointed if $\left|B_{0}\right|=1$, that is, there is a distinguished top vertex. The set of infinite paths, usually denoted $X$, is called the Bratteli compactum, and is a compact zero dimensional metric space, i.e., a compact Boolean space.

One can describe a Bratteli diagram using rectangular matrices with nonnegative integer entries. If $\left|B_{k}\right|=w(k)$ (with $w(0)=1$ ), define a matrix $M_{k}$ in $\mathbf{Z}^{w(k+1) \times w(k)}$ as follows. Index the vertices in $B_{k}$ by an initial segment of the positive integers, and set the $i, j$ entry to be the number of edges from point $j$ of $B_{k}$ to point $i$ of $B_{k+1}$. One may then form the limit dimension group, the direct limit, as ordered abelian groups,

$$
G_{B}=\lim \mathbf{Z}^{w(0)} \stackrel{M_{0}}{\longrightarrow} \mathbf{Z}^{w(1)} \stackrel{M_{1}}{\longrightarrow} \mathbf{Z}^{w(2)} \stackrel{M_{2}}{\longrightarrow} \cdots .
$$


Dimension groups may be defined in this fashion; it is clear how to recover the original Bratteli diagram. Note moreover, that there is a distinguished positive element, the image of the generator of the zeroth level, and this must be an order unit (a positive element of the dimension group such that every element of the dimension group is dominated by some multiple). A telescoping (also called "contraction" or "blocking") of a Bratteli diagram is the diagram obtained by telescoping the maps; replace $M_{0}$ by $M_{0}^{\prime}=M_{n(0)} M_{n(0)-1} \cdots M_{1} M_{0}$ : $\mathbf{Z}^{w(0)} \rightarrow \mathbf{Z}^{w(n(0)+1)}, M_{1}$ by $M_{1}^{\prime}=M_{n(1)} M_{n(1)-1} \cdots M_{n(0)+s} M_{n(0)+1}:$ $\mathbf{Z}^{w(n(0)+1)} \rightarrow \mathbf{Z}^{w(n(1)+1)}$, etc., where $\{n(0), n(1), \ldots\}$ is a strictly increasing sequence of positive integers.

We consider certain partial orderings on the path space. An ordered Bratteli diagram is a Bratteli diagram with a partial ordering in which (infinite) cofinal paths are linearly ordered, but two paths which are not cofinal are not comparable. One fairly general construction is the reverse lexicographic ordering, given in [HPS; Definition 2.3]. Suppose at each level there is a partial ordering on the edges, in which all the edges to the same vertex are linearly ordered, but edges with differing target vertices are not comparable. Then the finite paths, say from level $k$ to level $l$ (with $l>k$ ), can be ordered lexicographically. Then the ordering extends to cofinal paths (finite or infinite), as follows: if $x$ and $y$ have the same initial vertex, then $x>y$ if there exists $n$ such that $x_{n}>y_{n}$ and $x_{i}=y_{i}$ for all $i$ exceeding $n$. In particular, if two paths are comparable in this ordering, then they must be cofinal. Maximal and minimal paths are defined in the obvious manner. Telescopings of ordered Bratteli diagrams preserve the ordering on the infinite paths.

In [V], Vershik developed these ideas in order to obtain a map on the path space, defined except at the maximal paths, that simply sends each path to the smallest path exceeding it. Herman, Putnam and Skau considered the special case that there are unique maximal and unique minimal paths. They observe that in that case the map could be extended to a homeomorphism of the entire path space by sending the maximal path to the minimal path. In this case, we shall call it the lexicographic map (obtained from the ordering). They showed that every minimal homeomorphism of a zero dimensional compact metrizable space arises from this construction [HPS].

The orbits of a path under the original (Vershik) map consists of the paths cofinal to it; under the extended map, the orbit of the maximal path consists of everything cofinal with either it or the minimal path. 
Two spaces with homeomorphisms, $\left(X_{i}, \phi_{i}\right)(i=1,2)$ are orbit equivalent if there exists a homeomorphism $F: X_{1} \rightarrow X_{2}$ that sends orbits to orbits. When there is an orbit equivalence, for each point $x$ in $X_{1}$, there exists an integer $n(x)$ such that $F \phi_{1} x=\phi_{2}^{n(x)} F x$. This defines a function $n: X_{1} \rightarrow \mathbf{Z}$; similarly there is a function $m: X_{2} \rightarrow$ $\mathbf{Z}$ such that $F \phi_{1}^{m(x)} x=\phi_{2} F x$. Suppose that the spaces are compact and metrizable, and the homeomorphisms are topologically transitive (that is, have dense orbits). If $n$ is continuous, $\phi_{1}$ is topologically conjugate to $\phi_{2}$ or $\left(\phi_{2}\right)^{-1}[\mathbf{B}]$; if there are no periodic points, $n$ is continuous if it is bounded. The two homeomorphisms $\left(X_{1}, \phi_{1}\right)$ and $\left(X_{2}, \phi_{2}\right)$ are said to be strongly orbit equivalent if they are orbit equivalent, and the homeomorphism $F$ has associated cocycles $m$ and $n$ each with at most one point of discontinuity.

Giordano, Putnam, and Skau have shown that there is a strong orbit equivalence between the lexicographic maps if and only if the limit dimension groups with distinguished order unit (1.1) are order isomorphic. More recently, they also showed that the lexicographic maps are orbit equivalent if and only if the dimension groups are order isomorphic after factoring out their infinitesimal elements [GPS].

The dyadic odometer (or "adding machine") arises from the simple Bratteli diagram consisting of a single vertex and two edges at each level. There is (up to relabelling of the two edges at each level, which does not change the topological conjugacy class) just one ordering, and the lexicographic map is the adding machine. The limiting dimension group is obviously $\mathbf{Z}\left[\frac{1}{2}\right]$ with the natural ordering (as a subgroup of the reals), and with 1 in $Z\left[\frac{1}{2}\right]$ as the order unit. We exhibit examples of simple Bratteli diagrams with orderings (with unique maximal and minimal paths) whose lexicographic maps have arbitrary entropy, and for which the limit dimension groups are all $\mathbf{Z}\left[\frac{1}{2}\right]$. In particular, they are all (strongly) orbit equivalent to the adding machine. As a particular consequence, the natural question as to whether entropy is an invariant for orbit equivalence is answered negatively in drastic fashion.

We focus on the dimension group $\mathbf{Z}\left[\frac{1}{2}\right]$ for technical simplicity. We conjecture that any minimal homeomorphism of the Cantor set is strongly orbit equivalent to homeomorphisms of all entropies. In fact we conjecture something much stronger. If $T$ is a minimal homeomorphism of the Cantor set and $S$ is an ergodic measure-preserving isomorphism of nonatomic Lebesgue probability space, then there should exist a homeomorphism $T^{\prime}$ strongly orbit equivalent to $T$ 
and an invariant probability for $T^{\prime}$ with respect to which $T^{\prime}$ is measurably isomorphic to $S$. This would generalize Dye's Theorem [D] (all such $S$ are orbit equivalent in the measurable category). As orbit equivalent homeomorphisms have the same space of invariant Borel probabilities, the conjecture would also generalize Krieger's theorem ([K], see [DGS]) that every such $S$ is measurably isomorphic to some minimal uniquely ergodic homeomorphism of the Cantor set; every measurable system $S$ would be compatible with every space of invariant measures possible for minimal homeomorphisms of the Cantor set. These spaces are precisely the metrizable Choquet simplices, as follows from a theorem of Lazar and Lindenstrauss [LL] (a snappy proof of which can be deduced from [EHS; Theorem 2.2]) and the work of Herman, Putnam and Skau.

2. Finite entropy. We begin this section by showing that any Bratteli diagram can be telescoped so that the resulting diagram admits an ordering such that the corresponding lexicographic map has entropy zero. Then we construct lexicographic maps with arbitrary finite entropy, but with underlying dimension group $\mathbf{Z}\left[\frac{1}{2}\right]$; in particular, these lexicographic maps are all strongly orbit equivalent to the 2-adic adding machine, which of course has zero entropy.

We first recall subshifts and their entropies. Suppose that $A$ is a finite set. Let $A^{\mathrm{Z}}$ be the set of bisequences $x=\ldots x_{-1} x_{0} x_{1} \ldots$ (with each $x_{i}$ in $A$ ), equipped with the product topology. Then $A^{\mathrm{Z}}$ is a compact metrizable totally disconnected space, and the shift map $\sigma$ : $A^{\mathrm{Z}} \rightarrow A^{\mathrm{Z}}$ given by $(\sigma x)_{i}=x_{i+1}$ is a homeomorphism. The restriction of $\sigma$ to a closed shift invariant subset $Y$ of $A^{\mathrm{Z}}$ is a subshift. If $S$ is the restriction of $\sigma$ to $Y$, then the topological entropy of $S, h(S)$, is the growth rate of the number of words of length $n$ occurring in points of $Y$ :

$$
h(S)=\limsup _{n} \frac{\log \# \mathscr{W}_{n}(S)}{n}
$$

where $\mathscr{W}_{n}(S)=\left\{y_{1} y_{2} \cdots y_{n} \mid\right.$ there exists $y=\left(y_{i}\right)_{i \in \mathbf{Z}}$ in $\left.Y\right\}$. We suggest [Wa] or [DGS] as an introduction to topological entropy. This definition is given in [Wa; Theorem 7.13] or [DGS; Proposition 16.11].

If $\mathscr{W}$ is a set of words, let $S(\mathscr{W})$ be the subshift whose domain $Y$ is the set of all bisequences formed by concatenation of words in $\mathscr{W}$. For the purposes of this paper, it will suffice to assume that all words in $\mathscr{W}$ have the same length, say $l$, except in Proposition 2.2, below. Thus a bisequence $y$ is in $Y$ if and only if there is a bisequence 
$\ldots W\left(i_{-1}\right) W\left(i_{0}\right) W\left(i_{1}\right) \ldots$ and an integer $k$ with $0 \leq k<l$ such that for all $j$

$$
y_{k+j l} \cdots y_{k+(j+1) l-1}=W\left(i_{j}\right)
$$

LEMMA 2.1. Suppose $\mathscr{W}$ is a set consisting of $m$ distinct words of length $l$; form the subshift $S(\mathscr{W})$. Then

$$
h(S)=\frac{1}{l} \log m \text {. }
$$

Proof. For positive integers $k$ and $n$, suppose $k l \leq n<(k+1) l$. Then $m^{k} \leq \# \mathscr{W}_{n}(S) \leq l m^{(k+2)}$ (the last inequality holds because any word of length $n$ must be a subword of a concatenation of $k+2$ words from $\mathscr{W}$, beginning at one of the $l$ positions in the first word). Thus $\lim \log \left(\# \mathscr{W}_{n}(S)\right) / n$ exists and equals $\frac{1}{l} \log m$.

We establish some notation and parameters for this and the following section. Let $S$ be a lexicographic map on an ordered Bratteli compactum $X$. Let $\left\{1, \ldots, m_{k}\right\}$ represent and order $\mathscr{V}_{k}$, the set of vertices of level $k$. Let $A_{k}$ be the set of paths from $\mathscr{V}_{0}$ to $\mathscr{V}_{k}$. We use $\pi_{k}$ to denote any obvious truncation map, obtained by restriction of paths to their initial segments (that is, starting at $\mathscr{V}_{0}$ and truncating at $\left.\mathscr{V}_{k}\right)$. Given $k$, to each point $x$ in $X$, associate a bisequence $\left\{\pi_{k}\left(S^{n} x\right)\right\}$ in $A_{k}^{\mathrm{Z}}$, and let $S_{k}$ be the subshift with domain $\left\{\left\{\pi_{k}\left(S^{n} x\right)\right\} \mid x \in X\right\}$. (We remark that $S$ is the inverse limit of $\left\{S_{k}\right\}$.) Then

$$
h(S)=\lim _{k} h\left(S_{k}\right)
$$

(for example, [Wa; Theorem 7.6]).

An ordering on the path space of a Bratteli diagram is a consecutive ordering if whenever edges $u, v$, and $w$ have the same terminal vertex, $u$ and $w$ have the same initial vertex, and $u<v<w$, then $u$ and $v$ have the same initial vertex.

Proposition 2.2. Let $S$ be a lexicographic map of a Bratteli compactum with a consecutive ordering. Let $m_{k}$ be the number of vertices at level $k$ of the Bratteli diagram and let $n_{k}$ be the minimum number of arcs from a vertex at level $k-1$ to a vertex at level $k$. Suppose that

$$
\lim _{k \rightarrow \infty} \frac{\log \left(n_{k+1} \cdot m_{k}\right)}{n_{k+1}}=0 .
$$

Then $S$ has zero entropy. 
Proof. Recall that $S_{k}$ denotes the subshift which is the quotient of $S$ obtained by restricting to the first $k$ levels. A symbol in this subshift is a path from the top level to a vertex at level $k$. Suppose $u$ is a vertex at level $k$. Let $p_{1}, \ldots, p_{s}$ be the paths from the top level to $u$, listed in increasing order. Let $W(u)$ be the word of length $s$ which is the concatenation $p_{1} p_{2} \cdots p_{s}$. Now also assume that $v$ is a vertex at level $k+1$, that $a_{1}, \ldots, a_{t}$ are the edges from $u$ to $v$, and that $y$ is an infinite path in the Bratteli diagram such that $y_{1} \cdots y_{k+1}=p_{1} a_{1}$. If $y^{\prime}$ is the image of $y$ in $S_{k}$, then it follows that $y_{0}^{\prime} \cdots y_{s t-1}^{\prime}$ is the word $W(u)$ concatenated $t$ times. Therefore $S_{k}$ is contained in the subshift $T=S(\mathscr{W})$, where

$$
\mathscr{W}=\left\{(W(u))^{t} \mid u \text { is a vertex at level } k \text { where } n_{k+1} \leq t<2 n_{k+1}\right\} .
$$

If there is a length-decreasing bijection from $\mathscr{W}$ to a set $\mathscr{W}^{\prime}$ of distinct words, then $h\left(S\left(\mathscr{W}^{\prime}\right)\right) \geq h(S(\mathscr{W}))$. Let $\mathscr{W}^{\prime}$ be a collection of $m_{k} n_{k+1}$ distinct words of length $n_{k+1}$. Then

$$
h\left(S_{k}\right) \leq h(S(\mathscr{W})) \leq h\left(S\left(\mathscr{W}^{\prime}\right)\right)=\frac{\log \left(m_{k} n_{k+1}\right)}{n_{k+1}}
$$

where the equality follows from Lemma 2.1. Therefore $h(S)=$ $\lim h\left(S_{k}\right)=0$.

THEOREM 2.3. Any minimal homeomorphism of a Cantor set is strongly orbit equivalent to a homeomorphism of zero entropy.

Proof. By [HPS], we may assume without loss of generality that our homeomorphism is given as a lexicographic map $T$ defined by means of a nontrivial simple ordered pointed Bratteli diagram $B$. Given integers $t>s$, let $n(s, t)$ be the minimum number of paths from a vertex at level $s$ to a vertex at level $t$. Then for $s$ fixed, $n(s, t)$ goes to infinity as $t$ does. Therefore we may define recursively a sequence $\{j(k)\}$ such that if $B^{\prime}$ is the telescoped diagram whose vertex set at level $k$ is the vertex set of $B$ at level $j(k)$, then in the notation of Proposition 2.2, we have $\lim _{k} \log \left(m_{k} n_{k+1}\right) /\left(n_{k+1}\right)=0$.

The original map $T$ is conjugate to the lexicographic map $T^{\prime}$ determined from $B^{\prime}$ with the order induced from $B$. To define the desired zero entropy map $S$, we choose any consecutive ordering on $B^{\prime}$ whose minimal and maximal paths match those of $T^{\prime}$. Then $h(S)=0$ and $S$ is strongly orbit equivalent to $T$. 
Our entropy zero result above automatically yields the existence of strongly orbit equivalent minimal homeomorphisms with different entropies, independently of the examples in this section and $\S 3$, merely from the existence of minimal homeomorphisms of arbitrary entropy [DGS]. (Of course, it is interesting to construct examples explicitly, and moreover, examples strongly orbit equivalent to the odometer.)

Now we exhibit lexicographic maps of all possible finite entropies strongly orbit equivalent to the dyadic odometer. To this end, we make a special assumption, which has the effect of guaranteeing that the underlying dimension group is of rank one: Suppose that for $k \geq 1$, there are $n_{k}$ arcs from each vertex in $\mathscr{V}_{k-1}$ to each vertex $v$ in $\mathscr{V}_{k}$.

Let $l_{k}$ be the number of paths from the unique vertex in $\mathscr{V}_{0}$ to each vertex in $\mathscr{V}_{k}$, so $l_{k}=n_{k} m_{k-1} l_{k-1}$. Suppose that for each $v$ in $\mathscr{V}_{k+1}$ we have a linear ordering on the $m_{k} n_{k+1}$ arcs to $v$ from $\mathscr{V}_{k}$. This ordering determines an $m_{k} n_{k+1}$-tuple $\sigma_{v}$ with entries from $\left\{1,2, \ldots, m_{k}\right\}-\sigma_{v}(i)$ is the initial vertex in $\mathscr{V}_{k}$ of the $i$ th arc to $v$. We say the arcs to vertices of $\mathscr{V}_{k+1}$ have distinct orderings if for all $v$ and $v^{\prime}$ in $\mathscr{V}_{k+1}$,

$$
\sigma_{v}=\sigma_{v^{\prime}} \text { implies } v=v^{\prime} \text {. }
$$

For example, in Illustration 2.1 below, for the leftmost configuration, $\sigma_{v}=(3,2,1,2,1)$; for the middle one, $\sigma_{v}=(2,1,2,1,3)$, and for the rightmost one, $\sigma_{v}=(2,3,2,1,1)$.

In order to accommodate the unique maximal and minimal path condition, we additionally require for each $v$ in $\mathscr{V}_{k}$ that $\sigma_{v}(1)=1$ and $\sigma_{v}\left(m_{k} n_{k}\right)=m_{k}$ (note that none of the three examples in the illustration satisfy this!). As a technical convenience, we formulate the following condition:

(2.4) If $k \geq 1$ and $i$ and $j$ are (not necessarily distinct) vertices in $\mathscr{V}_{k}$, there is a vertex $v$ in $\mathscr{V}_{k+1}$ and $t<$ $m_{k} n_{k+1}$ such that $\sigma_{v}(t)=i$ and $\sigma_{v}(t+1)=j$.

The $l_{k}$ paths from $\mathscr{V}_{0}$ to a vertex $v$ in $\mathscr{V}_{k}$ are ordered as explained in $\S 1$. For $v$ at some level, say $k$, let $\mathscr{W}(v)$ denote the word of length

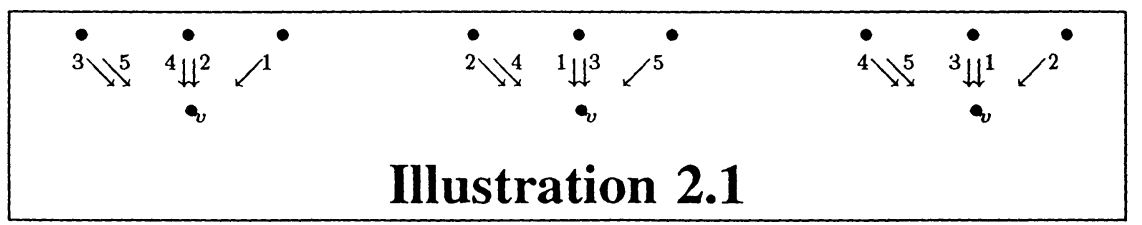


$l_{k}$ on symbols of $A_{k}$ whose $i$ th symbol is the $i$ th path from $\mathscr{V}_{0}$ to $v$. Let $S_{k}(t)=S(\mathscr{W})$ for the collection of words $\mathscr{W}=\left\{\pi_{k} \mathscr{W}(v) \mid v \in\right.$ $\left.\mathscr{V}_{k+t}\right\}$. So $S_{k}(t)$ is the subshift of $A_{k}^{\mathrm{Z}}$ generated by the truncations to the first $k$ levels of the paths of length $k+t$ starting at $\mathscr{V}_{0}$ (and terminating at $\mathscr{V}_{k+t}$ ).

LeMma 2.5. Let $S$ be a lexicographic map on an ordered Bratteli compactum as above, satisfying (2.4). Then for $k \geq 1$,

$$
h\left(S_{k}\right)=\lim _{t} h\left(S_{k}(t)\right) \text {. }
$$

Proof. Clearly $S_{k}(0) \supset S_{k}(1) \supset \cdots$ and $S_{k} \subset \bigcap_{t \geq 1} S_{k}(t)$. Conversely, suppose $y$ belongs to $\bigcap_{t \geq 1} S_{k}(t)$. By compactness, $y$ belongs to $S_{k}$ if for any positive integer $n$, the word $y_{-n} \cdots y_{n}$ appears in a point of $S_{k}$. It suffices to show $y_{-n} \cdots y_{n}$ occurs in the subshift generated by $\pi_{k} \mathscr{W}(v)$ for some vertex $v$.

Choose $i \geq k$ such that $l_{i}>2 n$. Since $y$ belongs to $S_{k}(i-k)$, $y_{-n} \cdots y_{n}$ is a subword of $\pi_{k} \mathscr{W}\left(v^{\prime}\right) \pi_{k} \mathscr{W}\left(v^{\prime \prime}\right)$ for some vertices $v^{\prime}$ and $v^{\prime \prime}$ in $\mathscr{V}_{i}$. By condition $(2.4), \pi_{k} \mathscr{W}\left(v^{\prime}\right) \pi_{k} \mathscr{W}\left(v^{\prime \prime}\right)$ is a subword of $\pi_{k} \mathscr{W}(v)$ for some $v$ in $\mathscr{V}_{i+1}$.

Therefore $S_{k}$ is the nested intersection of the subshifts $S_{k}(t)$. It is not hard to check that this implies $h\left(S_{k}\right)=\lim _{t} h\left(S_{k}(t)\right)$ [DGS; p. 113, Corollary to Proposition 16.16].

LEMMA 2.6. Let $S$ be a lexicographic map on an ordered Bratteli compactum as above, satisfying (2.4). Suppose that for all $k \geq 1$, the arcs from vertices of $\mathscr{V}_{k+1}$ have distinct orderings. Then

$$
h(S)=\lim _{k} \frac{\log m_{k}}{l_{k}}
$$

Proof. Suppose $k \geq 1$ and $t \geq 0$. Let $A_{k}^{*}$ denote the set of words which are concatenations of words in $A_{k}$. The hypothesis of distinct orderings implies that the map $\pi_{k}:\left\{\mathscr{W}(v) \mid v \in \mathscr{V}_{k+t}\right\} \rightarrow A_{k}^{*}$ is one to one. Therefore

$$
\begin{aligned}
h\left(S_{k}(t)\right) & =h\left(S_{k+t}(0)\right)=\frac{1}{l_{k+t}} \log m_{k+t}, \\
h(S) & =\lim _{k} h\left(S_{k}\right)=\lim _{k} \lim _{t} h\left(S_{k}(t)\right)=\lim _{k} \lim _{t} \frac{1}{l_{k+t}} \log m_{k+t},
\end{aligned}
$$

which finishes the proof. In fact, $h(S)=h\left(S_{k}\right)$ when $k \geq 1$. 
To use Lemma 2.6, we control the differences, $\frac{1}{l_{k}} \log m_{k}-$ $\frac{1}{l_{k+1}} \log m_{k+1}$. We require some notation to frame our estimates. Suppose $m$ and $n$ are integers exceeding 1 . Let $M(m, n)$ be the number of $m n$-tuples $\sigma$ whose entries are from $\{1,2, \ldots, m\}$ and satisfy the following conditions:

(i) $\sigma(1)=1$ and $\sigma(m n)=m$,

(ii) each element of $\{1,2, \ldots, m\}$ occurs exactly $n$ times in $\sigma$.

Let $M^{\prime}(m, n)$ be the largest power of two less than or equal $M(m, n)$. Obviously $M^{\prime}(m, n) \leq m^{m n}$.

LEMMA 2.7.

$$
\lim _{n}\left(\frac{M^{\prime}(m, n)}{m^{m n}}\right)^{1 / m n}=1
$$

Proof. We first note that $M^{\prime}(m, n) \geq \frac{1}{2} M(m, n)$, so it suffices to show the limit is 1 when $M(m, n)$ replaces $M^{\prime}(m, n)$. The number of $m n$-tuples on $\{1,2, \ldots, m\}$ satisfying (ii) above is $(m n) ! /(n !)^{m}$. The fraction of such in which 1 is the first entry and $m$ is the last is given by

$$
c(m, n)=\frac{(m n-2) !}{(m n) !}\left(\frac{(n-1) !}{n !}\right)^{2}=\frac{1}{(m n)(m n-1) n^{2}} .
$$

Clearly $c(m, n)^{1 / m n} \rightarrow 1$ as $n \rightarrow \infty$. So we are reduced to showing

$$
\lim _{n} \frac{\left((m n) ! /(n !)^{m}\right)^{1 / m n}}{m}=1 \text {. }
$$

The crude inequalities (valid for $N$ at least 2),

$$
(N / e)^{N}<N !<((N+1) / e)^{N+1}
$$

yield,

$$
\begin{aligned}
\frac{\left((m n) ! /(n !)^{m}\right)^{1 / m n}}{m} & >\frac{1}{m} \frac{(m n / e)}{((n+1) / e)((n+1) / e)^{1 / n}} \\
& =\frac{n}{n+1}\left(\frac{e}{n+1}\right)^{1 / n},
\end{aligned}
$$

which obviously goes to 1 as $n$ goes to infinity. The other inequality is similarly dealt with.

TheOREM 2.8. Suppose $0<\log \alpha<\infty$. There exists a homeomorphism $S$ strongly orbit equivalent to the dyadic adding machine such that $h(S)=\log \alpha$. 
Proof. By the comments in $\S 1$, we need only construct a lexicographic map whose underlying dimension group is $\mathbf{Z}\left[\frac{1}{2}\right]$. To this end, choose $m_{1}$ a power of 2 such that $\log m_{1}>\log \alpha$. Begin the construction of a Bratteli diagram by setting $\# \mathscr{V}_{0}=1, \mathscr{V}_{1}=\left\{1,2, \ldots, m_{1}\right\}$, and adjoining one edge to each vertex in $\mathscr{V}_{1}$ from $\mathscr{V}_{0}$. Now we describe the recursive step of the construction.

Begin with the ordered Bratteli diagram from $\mathscr{V}_{0}$ to $\mathscr{V}_{k}$ with $\frac{1}{l_{k}} \log m_{k}-\log \alpha=\varepsilon>0$. Pick a power of $2, n_{k+1}$, such that the following hold:

$$
\begin{gathered}
\left(M^{\prime}\left(m_{k}, n_{k+1}\right)^{1 / m_{k} n_{k+1}}\right)^{1 / l_{k}}>\alpha, \\
\frac{\log 2}{n_{k+1}}<\frac{\varepsilon}{2}, \\
\alpha^{l_{k+1}}>m_{k}^{2},
\end{gathered}
$$

where $l_{k+1}=n_{k+1} m_{k} l_{k}$; we can achieve (2.9) as a consequence of Lemma 2.7.

Next, choose a power of $2, m_{k+1}$, such that $m_{k+1} \leq M^{\prime}\left(m_{k}, n_{k+1}\right)$ and

This is possible since

$$
0<\frac{\log m_{k+1}}{l_{k+1}}-\log \alpha<\frac{\varepsilon}{2}
$$

$$
\frac{\log M^{\prime}\left(m_{k}, n_{k+1}\right)}{l_{k+1}}=\log M^{\prime}\left(m_{k}, n_{k+1}\right)^{1 / n_{k+1} m_{k} l_{k}}>\log \alpha \quad \text { (by 2.9) }
$$

and

$$
\frac{\log 2^{i+1}}{l_{k+1}}-\frac{\log 2^{i}}{l_{k+1}}=\frac{\log 2}{n_{k+1} m_{k} l_{k}}<\frac{\varepsilon}{2} \quad(\text { by } 2.10) .
$$

Now assign to each vertex $v$ of $\mathscr{V}_{k+1}$ an $m_{k} n_{k+1}$-tuple $\sigma_{v}$, determining the ordering of its incoming arcs. As $m_{k+1} \leq M^{\prime}\left(m_{k}, n_{k+1}\right)$, we can insist that $\sigma_{v}(1)=1$ and $\sigma_{v}\left(m_{k} n_{k+1}\right)=m$, and the map $v \mapsto \sigma_{v}$ be injective. By (2.11) and since $m_{k+1}>\alpha^{l_{k+1}}$, we have enough vertices in $\mathscr{V}_{k+1}$ to assign orderings of arcs which satisfy the technical condition (2.4).

This finishes the construction. Clearly $\lim _{k} \frac{1}{l_{k}} \log m_{k}=\log \alpha$ and by Lemma 2.6 , this equals $h(S)$. Finally, since the number of vertices $\left(m_{k}\right)$ at each level, and the multiplicity of arcs from each vertex to each vertex at the next level $\left(n_{k}\right)$ are all powers of 2 , the limiting dimension group is $\mathbf{Z}\left[\frac{1}{2}\right]$.

3. Infinite entropy. In this section, we construct an infinite entropy lexicographic map $S$ strongly orbit equivalent to the dyadic adding machine. This is an elaboration of the finite entropy construction. As 
in $\S 2, m_{k}$ is the number of vertices at level $k, n_{k}$ is the number of arcs to each vertex in $\mathscr{V}_{k}$ from each vertex in $\mathscr{V}_{k-1}$, and $l_{k}$ is the number of paths from $\mathscr{V}_{0}$ to each vertex in $\mathscr{V}_{k}$. In addition, $\mathscr{V}_{k}$ will be the disjoint union of sets $\mathscr{V}_{k}(t), 1 \leq t \leq j_{k}$ each of cardinality $\overline{m_{k}}$. The construction is recursive.

We begin with $m_{1}=n_{1}=2$ and $j_{1}=1$. Given the ordered graph from $\mathscr{V}_{0}$ through $\mathscr{V}_{k}$, we construct $\mathscr{V}_{k+1}$ and the ordered arcs from $\mathscr{V}_{k}$ to $\mathscr{V}_{k+1}$; we choose $n_{k+1}$, let $\bar{m}_{k+1}=M^{\prime}\left(m_{k}, n_{k+1}\right)$, and to each of the $\bar{m}_{k+1}$ vertices $v$ in $\mathscr{V}_{k+1}(1)$ assign a distinct ordering of the $m_{k} n_{k+1}$ arcs from $\mathscr{V}_{k}$ to $v$, with each ordering beginning at the first vertex of $\mathscr{V}_{k}$ (for some arbitrary linear ordering on $\mathscr{V}_{k}$ ) and ending at the last vertex of $\mathscr{V}_{k}$. This determines $l_{k+1}=n_{k+1} l_{k} m_{k}$. Then choose $j_{k+1}$ with $m_{k+1}=j_{k+1} \bar{m}_{k+1}$ such that $\lim _{k} \frac{1}{l_{k+1}} \log j_{k+1} \bar{m}_{k+1}=\infty$.

For each $t, 2 \leq t \leq j_{k+1}$, we assign the same set of arc orderings to $\mathscr{V}_{k+1}(t)$ as were assigned to $\mathscr{V}_{k+1}(1)$. We require $n_{k+1}$ and $j_{k+1}$ to be powers of 2 , so $S$ will be strongly orbit equivalent to the dyadic adding machine.

The basic idea is that if the numbers $n_{k}$ are large enough, then $h\left(S_{k}\right)$ is not much less than $\frac{1}{k} \log m_{k}$, so $h(S)=\lim _{k} h\left(S_{k}\right)=\infty$. To carry this out, we first specify the parameters at the recursive step. Define positive numbers $\varepsilon_{k}$ by $\prod_{i=1}^{k}\left(1-\varepsilon_{i}\right)=\frac{1}{2}+2^{-k-1}$, so

$$
\prod_{i=1}^{\infty}\left(1-\varepsilon_{i}\right)=\frac{1}{2} \text {. }
$$

Suppose we are given the ordered Bratteli diagram from $\mathscr{V}_{0}$ through $\mathscr{V}_{k}$. By Lemma 2.7, we may choose a power of $2, n_{k+1}$ such that

$$
\left(M^{\prime}\left(m_{k}, n_{k+1}\right)\right)^{1 / m_{k} n_{k+1}}>\left(1-\varepsilon_{k+1}\right) m_{k} .
$$

Now $\bar{m}_{k+1}=M^{\prime}\left(m_{k}, n_{k+1}\right), l_{k+1}=n_{k+1} l_{k} m_{k}$, and

$$
\left(\bar{m}_{k+1}\right)^{1 / l_{k+1}}>\left(1-\varepsilon_{k+1}\right)^{1 / l_{k}}\left(m_{k}\right)^{1 / l_{k}} \text {. }
$$

Then choose a power of $2, j_{k+1}$, such that $\left(j_{k+1}\right)^{1 / l_{k+1}}>2$. Now $m_{k+1}=\bar{m}_{k+1} j_{k+1}$. This finishes the construction.

Now we observe that

$$
\begin{aligned}
h\left(S_{k+1}(0)\right) & =\frac{1}{l_{k+1}} \log m_{k+1}=\frac{1}{l_{k+1}} \log \bar{m}_{k+1} j_{k+1} \\
& =\log \bar{m}_{k+1}^{1 / l_{k+1}}+\log j_{k+1}^{1 / l_{k+1}} \\
& >\log \left[\left(1-\varepsilon_{k+1}\right) m_{k}^{1 / l_{k}}\right]+\log j_{k+1}^{1 / l_{k+1}} \\
& >h\left(S_{k}(0)\right)+\log 2+\log \left(1-\varepsilon_{k+1}\right) .
\end{aligned}
$$

Therefore $\lim _{k} h\left(S_{k}(0)\right)=\infty$. 
Next, given $k \geq 1$ and $t \geq 1$, let

$$
J=\left(\prod_{i=1}^{t} j_{k+i}^{1 / l_{k+i}}\right)^{l_{k+t}}
$$

We claim

$$
\#\left\{\pi_{k} W(v) \mid v \in \mathscr{V}_{k+t}\right\} \geq \frac{1}{J} m_{k+t} .
$$

To prove (3.3), it suffices to show that the map

$$
\begin{aligned}
\left\{W(v) \mid v \in \mathscr{V}_{k+t}\right\} & \rightarrow\left\{W(v) \mid v \in \mathscr{V}_{k}\right\}^{*} \\
W & \mapsto \pi_{k}(W)
\end{aligned}
$$

is at most $J$ to one. To this end, we remark that for each $v$ in $\mathscr{V}_{k+t}$, for each $i$ between 1 and $t$, there are vertices $v_{r} \quad(1 \leq r \leq a(i))$ such that $\pi_{k+i}(W(v))$ is a concatenation $W\left(v_{1}\right) \cdots W\left(v_{a(i)}\right)$, where $v_{r}$ belongs to $\mathscr{V}_{k+i}\left(t_{r}\right)$ and $a(i)=l_{k+t} / l_{k+i}$. Given $i$, the number of possible sequences $t_{1} \cdots t_{a(i)}$ is $j_{k+i}^{a(i)}$. The number of ways to fix these sequences for all $i$ with $1 \leq i \leq t$ is at most $\prod_{i=1}^{t} j_{k+i}^{a(i)}=J$.

However, given one of these $J$ choices, and given $\pi_{k}(W(v))$, we can recover $W(v)$, since $\pi_{k}(W(v))$ and the sequence $t_{r}$ for $i=1$ determine $\pi_{k+1}(W(v))$, by the distinctness of the arc orderings chosen for $\mathscr{V}_{k+1}(1)$; similarly, we recover $\pi_{k+2}(W(v)), \ldots, \pi_{k+t}(W(v))=$ $W(v)$. This proves (3.3).

Finally, suppose $k, t \geq 1$. Then

$$
\begin{aligned}
h\left(S_{k}(t)\right) & =\frac{1}{l_{k+t}} \log \left(\#\left\{\pi_{k}(W(v)) \mid v \in \mathscr{V}_{k+t}\right\}\right) \\
& \geq \frac{1}{l_{k+t}} \log \frac{m_{k+t}}{J} \quad(\text { by } 3.3)
\end{aligned}
$$

and therefore

$$
h\left(S_{k}(t)\right) \geq \log \left(\frac{m_{k+t}}{J}\right)^{1 / l_{k+t}}
$$

By (3.2), we have

$$
\begin{aligned}
m_{k+t}^{1 / l_{k+t}} & =j_{k+t}^{1 / l_{k+t}} \bar{m}_{k+t}^{1 / l_{k+t}} \\
& >j_{k+t}^{1 / l_{k+t}}\left(1-\varepsilon_{k+t}\right)^{1 / l_{k+t-1}} m_{k+t-1}^{1 / l_{k+t-1}} \\
& >\cdots>\left(\prod_{i=1}^{t} j_{k+i}^{1 / l_{k+t}}\right)\left(\prod_{i=1}^{t}\left(1-\varepsilon_{k+i}\right)^{1 / l_{k+1-1}}\right) m_{k}^{1 / l_{k}}
\end{aligned}
$$


Therefore

Substituting this into (3.4) yields

$$
m_{k+t}^{1 / l_{k+t}}>J^{1 / l_{k+t}} \cdot \frac{1}{2} \cdot m_{k}^{1 / l_{k}} .
$$

$$
h\left(S_{k}(t)\right)>h\left(S_{k}(0)\right)-\log 2 ;
$$

thus $h(S)=\lim _{k} \lim _{t} h\left(S_{k}(t)\right)=\infty$.

\section{REFERENCES}

[B] M. Boyle, Topological orbit equivalence and factor maps in symbolic dynamics, $\mathrm{Ph} . \mathrm{D}$. Thesis, University of Washington, Seattle, 1983.

[DGS] M. Denker, C. Grillenberger and K. Sigmund, Ergodic Theory on Compact Spaces, Lecture Notes in Math., vol. 527, Springer-Verlag, 1976.

[D] H. Dye, On groups of measure preserving transformations, Amer. J. Math., 81 (1959), 119-159.

[EHS] E. G. Effros, D. E. Handelman and C-L. Schen, Dimension groups and their affine representations, Amer. J. Math., 102 (1980), 385-407.

[GPS] T. Giordano, I. F. Putnam and C. F. Skau, Topological orbit equivalence and $C^{*}$ crossed products, in preparation.

[HPS] R. H. Herman, I. F. Putnam and C. F. Skau, Ordered Bratteli diagrams, dimension groups, and topological dynamics, Internat. J. Math., 3 (1992), 827-864.

[K] W. Krieger, On unique ergodicity, Proc. of the Sixth Berkeley Symposium on Mathematics, Statistics, and Probability, Berkeley and Los Angeles, University of California Press, 1 (1972), 327-346.

[LL] A. J. Lazar and J. Lindenstrauss, Banach spaces whose dual are $L_{1}$ spaces and their representing matrices, Acta Math., 126 (1971), 165-193.

[P] I. F. Putnam, The $C^{*}$-algebras associated with minimal homeomorphisms of the Cantor set, Pacific J. Math., 136 (1989), 329-353.

[Sk] C. Skau, Minimal dynamical systems, ordered Bratteli diagrams and associated $C^{*}$-crossed products, (preprint), Proceedings Nara Conference, 1991.

[V] A. M. Vershik, Uniform algebraic approximation of shift and multiplication operators, Soviet Math. Dokl., 24 (1) (1981), 97-100.

[Wa] P. Walters, An Introduction to Ergodic Theory, Graduate Texts in Mathematics, vol. 79, Springer-Verlag, 1981.

Received March 25, 1992 and in revised form March 20, 1993. The first author was partially supported by NSF, and the second author was partially supported by an operating grant from NSERC (Canada).

UNIVERSITY OF MARYLAND

College Park, MD 20742

E-mail address: mmb@math.umd.edu

AND

UNIVERSITY OF OTTAWA

OtTawa, Ontario, K1N 6N5

CANADA

E-mail address: dehsg@ACADVM1.uottawa.ca 



\section{PACIFIC JOURNAL OF MATHEMATICS}

Founded by

E. F. BeCKenbach (1906-1982) F. Wolf (1904-1989)

\section{EDITORS}

Sun-Yung A. Chang

(Managing Editor)

University of California

Los Angeles, CA 90024-1555

chang@math.ucla.edu

\section{F. Michael Christ}

University of California

Los Angeles, CA 90024-1555

christ@math.ucla.edu

\section{Herbert Clemens}

University of Utah

Salt Lake City, UT 84112

clemens@math.utah.edu

\author{
THOMAS ENRIGHT \\ University of California, San Diego \\ La Jolla, CA 92093 \\ tenright@ucsd.edu \\ Nicholas ERcolanI \\ University of Arizona \\ Tucson, AZ 85721 \\ ercolani@math.arizona.edu \\ R. FINN \\ Stanford University \\ Stanford, CA 94305 \\ finn@gauss.stanford.edu \\ VAUGHAN F. R. JONES \\ University of California \\ Berkeley, CA 94720 \\ vfr@math.berkeley.edu
}

Steven KerckhofF

Stanford University

Stanford, CA 94305

spk@gauss.stanford.edu

Martin SCHARLEMANN

University of California

Santa Barbara, CA 93106

mgscharl@math.ucsb.edu

Harold Stark

University of California, San Diego

La Jolla, CA 92093

V. S. VARADARAJAN

University of California

Los Angeles, CA 90024-1555

vsv@math.ucla.edu

\section{SUPPORTING INSTITUTIONS}

\author{
UNIVERSITY OF ARIZONA \\ UNIVERSITY OF BRITISH COLUMBIA \\ CALIFORNIA INSTITUTE OF TECHNOLOGY \\ UNIVERSITY OF CALIFORNIA \\ UNIVERSITY OF MONTANA \\ UNIVERSITY OF NEVADA, RENO \\ NEW MEXICO STATE UNIVERSITY \\ OREGON STATE UNIVERSITY
}

UNIVERSITY OF OREGON

UNIVERSITY OF SOUTHERN CALIFORNIA

STANFORD UNIVERSITY

UNIVERSITY OF HAWAII

UNIVERSITY OF UTAH

WASHINGTON STATE UNIVERSITY

UNIVERSITY OF WASHINGTON 


\section{PACIFIC JOURNAL OF MATHEMATICS}

Volume $164 \quad$ No. $1 \quad$ May 1994

Entropy versus orbit equivalence for minimal homeomorphisms

M. MiChaEL BoYle and DAVID E. HANDELMAN

The hyperspaces of infinite-dimensional compacta for covering and cohomological dimension are homeomorphic

TADEUSZ WLADYSLAW DOBROWOLSKI and LEONARD RUBIN

The index of transversally elliptic operators for locally free actions

JefFrey Stephen Fox and Peter Evarts Haskell

Unit indices of some imaginary composite quadratic fields

MiKiHITO HIRABAYASHI

Periodic points on nilmanifolds and solvmanifolds

EDWARD KEPPELMANN

Branched coverings of surfaces with ample cotangent bundle

MiCHAEL JEROME SPURR

Evolutionary existence proofs for the pendant drop and $n$-dimensional 147 catenary problems

ANDREW GUY STONE

Lattices of Lipschitz functions

NiKOLAI ISAAC WEAVER

Correction to: "Trace rings for verbally prime algebras" 